\title{
MODELO APLICADO À DINÂMICA DA ÁGUA E DO POTÁSSIO NO SOLO SOB IRRIGAÇÃO POR GOTEJAMENTO - ANÁLISE DE SENSIBILIDADE
}

\author{
RENÉ N.C. RIVERA ${ }^{1}$, JARBAS H. MIRANDA ${ }^{2}$, SÉRGIO N. DUARTE ${ }^{3}$, \\ TARLEI A. BOTREL ${ }^{4}$
}

\begin{abstract}
RESUMO: A compreensão do transporte simultâneo da água e de solutos, a partir de uma fonte pontual, permite desenvolver estratégias eficientes na fertirrigação, sendo importante no dimensionamento, na operação e no manejo de sistemas de irrigação localizada. O presente trabalho teve como objetivo apresentar a análise de sensibilidade de um modelo matemático, desenvolvido para simular o deslocamento simultâneo de água e de potássio na irrigação por gotejamento. A análise de sensibilidade foi realizada com respeito às variações individuais da condutividade hidráulica do solo saturado, umidade inicial e saturada do solo, dispersividade, fator de retardamento e vazão do emissor. Os resultados obtidos revelaram que o modelo matemático, com relação à distribuição de água, é bastante sensível à umidade de saturação e inicial do solo e, em relação à distribuição de potássio, é bastante sensível a variações negativas da condutividade hidráulica do solo saturado e da vazão do gotejador; já em relação aos parâmetros de transporte de solutos no solo, é bastante sensível a variações negativas do fator de retardamento e pouco sensível às variações da dispersividade do solo.
\end{abstract}

PALAVRAS-CHAVE: deslocamento miscível, dinâmica de solutos, modelagem computacional.

\section{MODEL APPLIED TO WATER AND POTASSIUM DYNAMICS UNDER DRIP IRRIGATION - SENSITIVITY ANALYSIS}

\begin{abstract}
The understanding of the simultaneous transport of water and solute, from a punctual source, allows developing efficient strategies in fertigation, being important in design, operation and management of drip irrigation. The present work had as objective to show the sensitivity analysis of a mathematical model, developed to simulate the simultaneous water and potassium movement in drip irrigation. The sensitivity analysis was performed considering individual variations of saturated soil hydraulic conductivity, initial and saturated soil moisture, dispersivity, retardation factor and emitter flow. The obtained results showed that the mathematical model, regarding water distribution, is quite sensitive to the saturation and initial soil moisture. In relation to potassium distribution, it is quite sensitive to negative variations of soil saturated hydraulic conductivity, emitter flow and retardation factor of the soil, and less sensitive to the soil dispersivity variations.
\end{abstract}

KEYWORDS: miscible displacement, solute dynamics, computational modeling

\section{INTRODUÇÃO}

O declínio na disponibilidade de água de boa qualidade para irrigação é um problema crescente para a agricultura irrigada, devido, principalmente, ao incremento da competição com o uso em áreas urbanas e industriais. Assim, a irrigação por gotejamento é uma das tecnologias que se estão expandindo mais rapidamente na agricultura irrigada moderna, tendo em vista seu grande potencial para melhorar a economia do uso da água.

\footnotetext{
${ }^{1}$ Eng $^{\mathrm{o}}$ Agrônomo, Prof. Doutor, Universidad Mayor de San Andrés, La Paz, Bolivia, Calle Héroes del Acre, 1850, Fone: (5912) 249.1558,renechipana@yahoo.com

${ }^{2}$ Prof. Dr., Departamento de Ciências Exatas, ESALQ/USP, Piracicaba - SP, jhmirand@esalq.usp.br

${ }^{3}$ Prof. Dr., Departamento de Engenharia Rural, ESALQ/USP, Piracicaba - SP, snduarte@esalq.usp.br.

${ }^{4}$ Prof. Associado, Departamento de Engenharia Rural, ESALQ/USP, Piracicaba - SP, tabotrel@esalq.usp.br.

Recebido pelo Conselho Editorial em: 8-5-2007
}

Aprovado pelo Conselho Editorial em: 15-7-2008 
Os produtos químicos aplicados por meio da água de irrigação sofrem mudanças espacial e temporal no solo, variando sua distribuição no perfil, o que resulta em diferentes padrões de distribuição (MMOLAWA \& OR, 2000). Para CLOTHIER (1984), a compreensão do transporte simultâneo de água e de solutos, em duas ou três dimensões, a partir de uma fonte pontual, permite desenvolver estratégias eficientes, sendo importante no dimensionamento, na operação e no manejo da fertirrigação.

Os altos custos envolvidos nas pesquisas de campo e os avanços computacionais estão fazendo com que os modelos matemáticos, aliados às técnicas numéricas, se constituam em ferramentas bastante viáveis, possibilitando predição do destino e do comportamento da água e dos solutos na irrigação localizada.

As primeiras representações simplificadas do transporte de água e de solutos no solo foram feitas para prover estimativas grosseiras da lixiviação de materiais solúveis. Num segundo momento, modelos mais complexos têm sido desenvolvidos, tentando integrar os mecanismos físicos e químicos que influenciam no movimento de solutos. Esses modelos são baseados, geralmente, em soluções que utilizam métodos numéricos e usam a predição do fluxo de água como partida para a simulação do fluxo de solutos (WAGENET, 1986).

O desenvolvimento de modelos matemáticos para descrever com precisão o transporte de água e solutos é tarefa laboriosa, quando usados para condições reais de campo, pois o meio apresenta grande complexidade. Segundo GENUCHTEN \& WIERENGA (1986), vários modelos teóricos têm sido desenvolvidos ao longo dos anos para descrever o transporte de solutos no solo. $\mathrm{O}$ sucesso desses modelos, porém, depende, em grande parte, da capacidade de quantificar os parâmetros de transporte, que são as variáveis de entrada para esses modelos. Os parâmetros mais importantes são o fluxo do fluido, o coeficiente de dispersão e o fator de retardamento.

Segundo GENUCHTEN \& WIERENGA (1986), devido ao fato de o solo ser um meio poroso com extensa heterogeneidade química e física, a troca de solutos entre os poros de diferentes categorias pode ser dada por três vias: transporte de massa ou fluxo convectivo, transporte difusivo e transporte dispersivo.

O fator de retardamento $(\mathrm{Fr})$ pode ser definido como a capacidade de retenção ou o efeito "buffer" de um determinado elemento, ou como a velocidade do soluto em relação à velocidade da solução no poro (MATOS et al., 1999). Se não existirem interações entre o soluto e o solo, o valor do coeficiente de partição $(\mathrm{Kd})$ é zero, e o fator de retardamento torna-se unitário. Se Fr for menor que um, significa que apenas uma fração da fase líquida participa no processo de transporte, como ocorre, por exemplo, em agregados muito densos contendo poros de diâmetro muito pequeno (GENUCHTEN \& WIERENGA, 1986). Segundo TORIDE et al. (1999), existe correlação negativa entre a velocidade da solução no poro (v) e o coeficiente de distribuição ou de partição (Kd), o que implica que Fr e v também estão inversamente correlacionados. Assim, os parâmetros v e $\mathrm{Fr}$ determinam a forma com que o soluto avança ou é retido no solo (ALVAREZ, 1995).

As abordagens dos modelos de transporte de solutos variam em suas suposições, complexidade e também na necessidade de aquisição de dados de entrada. Segundo JURY (1984), os modelos de transporte de solutos no campo geralmente podem ser divididos em duas categorias: modelos determinísticos e modelos estocásticos. Os modelos determinísticos usam equações contínuas com parâmetros que variam em cada ponto no espaço e tem relação lógica fixa entre um ponto e o outro. Por outro lado, os modelos estocásticos usam parâmetros que podem variar fortuitamente e são caracterizados em termos de distribuição de probabilidade.

As dificuldades no uso de modelos estocásticos no dimensionamento de sistemas de irrigação é que esses não são prontamente adaptáveis a solos estratificados, requerendo extensa habilidade matemática para sua resolução.

A técnica do uso de modelos numéricos (volumes de controle, diferenças finitas e elementos finitos) permite que se trabalhe com facilidade em solos compostos por múltiplas camadas (CRUZ, 2000; TULI \& HOPMANS, 2004). 
Diante do exposto, o presente trabalho teve como objetivo apresentar e realizar a análise de sensibilidade de modelo matemático numérico e determinístico, desenvolvido para simular o deslocamento simultâneo de água e de potássio no solo sob irrigação por gotejamento, em relação aos seus principais parâmetros de entrada.

\section{MATERIAL E MÉTODOS}

O modelo computacional desenvolvido, denominado "Programa para o Transporte de Água e Solutos sob Irrigação por Gotejamento" (PTASIG), tem a característica de ser estruturado e apresentar várias opções, como a entrada de dados, criação de base de dados, recuperação de dados do disco e saída de dados, tanto na tela como na impressora.

Os valores de entrada do modelo são: vazão, tempo de irrigação, tempo de aplicação do soluto, tempo de redistribuição, incremento do tempo, dados do solo para cada camada (umidade inicial, umidade residual, umidade a saturação, profundidade do solo, raio de influência do emissor e parâmetros da equação de Genuchten) e dados do soluto (concentração inicial no solo, concentração na água de irrigação, coeficiente de difusão em água pura, fator de retardamento e dispersividade do solo).

\section{Desenvolvimento do modelo}

O desenvolvimento do modelo foi baseado na solução numérica das equações diferenciais parciais (EDP) de segunda ordem, aplicadas a fontes puntiformes sob fluxo transiente, permitindo determinar a distribuição simultânea de água e de solutos no solo, em função do espaço e do tempo. A eq.(1) representa a EDP utilizada para descrever a dinâmica do soluto no solo.

$$
\operatorname{Fr} \frac{\partial \theta \mathrm{C}}{\partial \mathrm{t}}=-\frac{\partial(\mathrm{qC})}{\partial \mathrm{z}}+\frac{\partial}{\partial \mathrm{z}}\left[\mathrm{D} \theta \frac{\partial \mathrm{C}}{\partial \mathrm{z}}\right]-\frac{\partial(\mathrm{qC})}{\partial \mathrm{x}}+\frac{\partial}{\partial \mathrm{x}}\left[\mathrm{D} \theta \frac{\partial \mathrm{C}}{\partial \mathrm{x}}\right]
$$

em que,

Fr - fator de retardamento, adimensional;

$\theta$ - umidade atual do solo, $\mathrm{L}^{3} \mathrm{~L}^{-3}$;

D - coeficiente de dispersão-difusão, adimensional;

q - volume da solução que passa em uma unidade de área de solo na unidade de tempo, $\mathrm{L} \mathrm{T}^{-1}$, e

$\mathrm{C}$ - massa do soluto por unidade de volume de solução, $\mathrm{ML}^{-3}$.

A equação básica que governa o movimento da água em solo não-saturado em regime de fluxo transitório é deduzida por meio do princípio da conservação da massa, podendo ser expressa pela eq.(2), que representa a taxa de acumulação de água por unidade de tempo t, no elemento de volume $\Delta \mathrm{V}$, nas coordenadas $\mathrm{x}$ e $\mathrm{z}$ (fluxo bidimensional), denominada também de equação da continuidade (LIBARDI, 2005):

$$
\frac{\partial \theta}{\partial t}=-\left(\frac{\partial q x}{\partial x}+\frac{\partial q z}{\partial z}\right)
$$

em que,

$\mathrm{t}$ - tempo, $\mathrm{T}$;

qx, qz - densidades de fluxo nas direções x e z, $\mathrm{L} \mathrm{T}^{-1}$, e

$\mathrm{x}, \mathrm{z}$ - direções, L.

Se na eq.(2) as densidades de fluxo qx e qz forem substituídas pela equação de DarcyBuckingham para as respectivas direções, obtém-se a eq.(3), que é a equação diferencial que governa o movimento de água em solos não-saturados, em regime transiente, também conhecida como equação de Richards (PREVEDELLO, 1996; LIBARDI, 2005).

$$
\frac{\partial \theta}{\partial \mathrm{t}}=\frac{\partial}{\partial \mathrm{x}}\left[\mathrm{Kx}(\theta) \frac{\partial \mathrm{H}}{\partial \mathrm{x}}\right]+\frac{\partial}{\partial \mathrm{z}}\left[\mathrm{Kz}(\theta) \frac{\partial \mathrm{H}}{\partial \mathrm{z}}\right]
$$


em que,

$\mathrm{Kx}, \mathrm{z}(\theta)$ - condutividade hidráulica do solo saturado, nas direções $\mathrm{x}$ e $\mathrm{z}, \mathrm{L} \mathrm{T}^{-1}$, e

$\mathrm{H}$ - potencial hidráulico total da água no solo, $\mathrm{L}$.

A solução foi implementada utilizando a técnica dos volumes de controle finitos, efetuando incrementos de espaço no sentido radial e vertical, e incrementos de tempo, levando-se em consideração as hipóteses descritas por BOTREL (1988) e CRUZ (2000), sendo a região de estudo assumida como tendo forma cilíndrica e composta de anéis concêntricos (Figura 1).

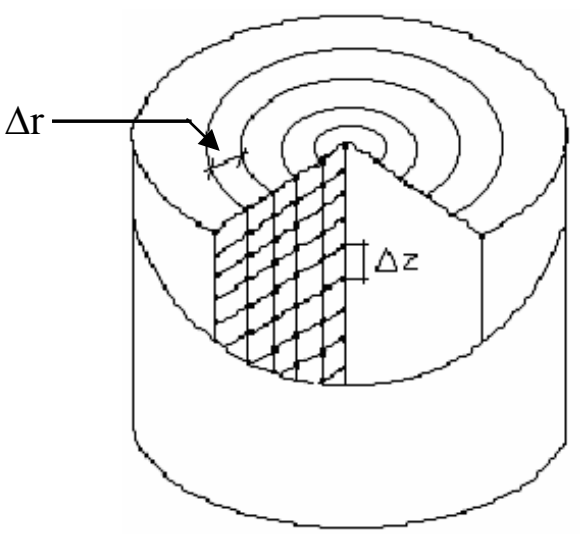

FIGURA 1. Esquema dos anéis concêntricos considerados na simulação para descrever a forma de um bulbo úmido hemisférico. Ring infiltrometer scheme considered in the simulation to describe the hemispheric wet bulb shape.

\section{Determinação da densidade do fluxo de água e de solutos}

O cálculo da densidade de fluxo de uma célula para outra foi realizado seguindo as considerações preconizadas por VAN DER PLOEG \& BENECKE (1974) e GENUCHTEN (1980), por meio das quais se assume que o fluxo dentro dos anéis ou células ocorre em duas direções, tanto para a entrada como para a saída de água e de solutos (Figura 2).

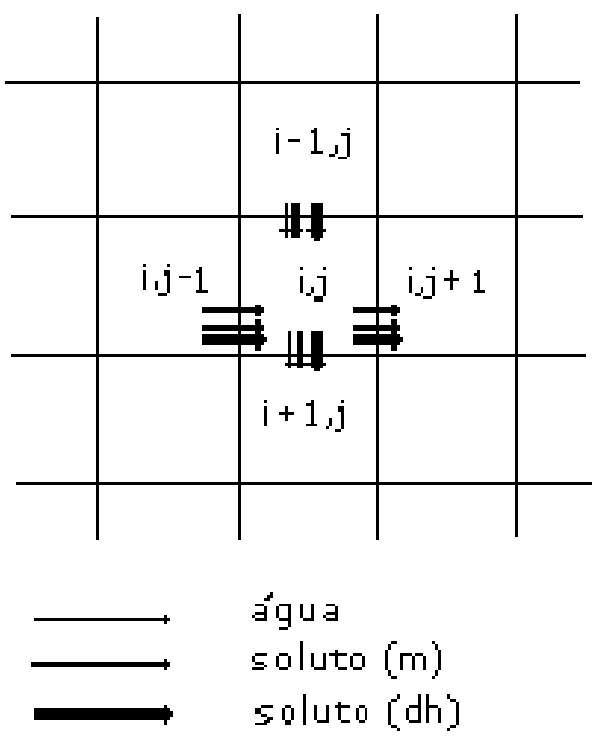

FIGURA 2. Coordenadas de células adjacentes necessárias ao cálculo do movimento da água e dos solutos por convecção $(\mathrm{m})$ e dispersão hidrodinâmica $(\mathrm{dh})$, nas direções radial e vertical. Coordinates of adjacent cells necessary to calculate the water flow and solute transport by convection (m) and hydrodynamic dispersion coefficient (dh), in the radial and vertical directions. 
Quando se aplica a técnica de discretização em volumes de controle, a eq.(1) pode ser reescrita como sendo eq.(4):

$$
\operatorname{Fr} \frac{\Delta \theta \mathrm{C}}{\Delta \mathrm{t}}=-\frac{\Delta(\mathrm{qC})}{\Delta \mathrm{z}}+\frac{\Delta}{\Delta \mathrm{z}}\left[\mathrm{D} \bar{\theta} \frac{\Delta \mathrm{C}}{\Delta \mathrm{z}}\right]-\frac{\Delta(\mathrm{qC})}{\Delta \mathrm{x}}+\frac{\Delta}{\Delta \mathrm{x}}\left[\mathrm{D} \bar{\theta} \frac{\Delta \mathrm{C}}{\Delta \mathrm{x}}\right]
$$

Ao se considerar a discretização em volumes de controle, a eq.(3) fica transformada na eq.(5):

$$
\frac{\Delta \theta}{\Delta \mathrm{t}}=-\overline{\mathrm{K}} \mathrm{x}(\theta) \frac{\Delta \mathrm{Hx}}{\Delta \mathrm{x}}-\overline{\mathrm{K}} \mathrm{z}(\theta) \frac{\Delta \mathrm{Hz}}{\Delta \mathrm{z}}
$$

Dessa maneira, inicialmente, o programa calcula para cada $\Delta \mathrm{t}$ (num volume do solo com limites $\Delta \mathrm{z}$ e $\Delta \mathrm{r}$ ) a saturação efetiva, o potencial da água no solo e a condutividade hidráulica (K). Com base nesses dados, determinam-se, para cada uma das células, a densidade de fluxo, a variação do volume de água, a umidade posterior do solo, o fluxo em massa de solutos e o fluxo por dispersão hidrodinâmica para, finalmente, por meio de balanço de massa, determinar a nova concentração do soluto no final de $\Delta \mathrm{t}$. Maiores detalhamentos do modelo podem ser verificados em RIVERA (2004) e RIVERA et al. (2006) (Figura 3).

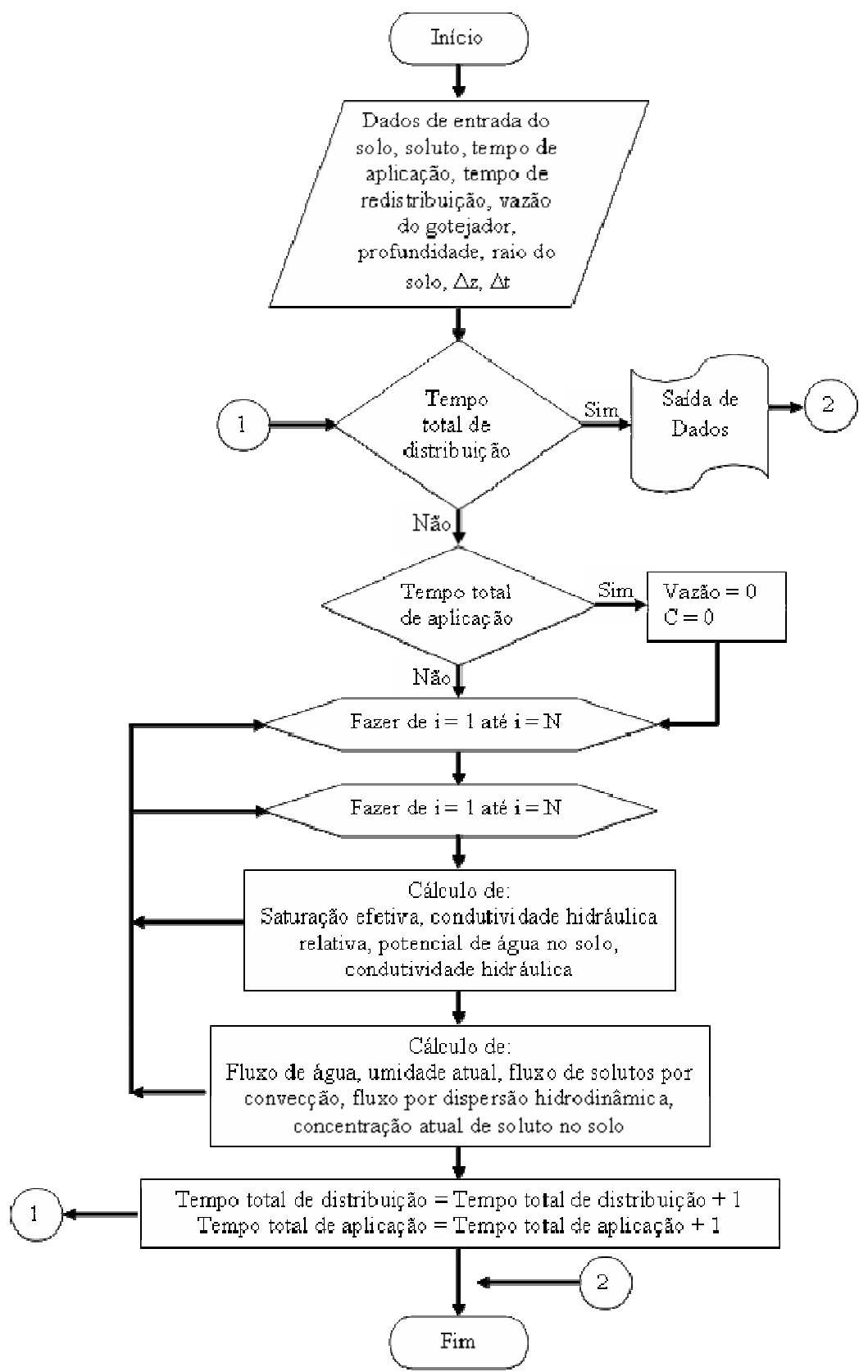

FIGURA 3. Fluxograma do modelo proposto. Flowchart of the proposed model. 


\section{Parâmetros físico-hídricos do solo}

Em relação ao material de solo empregado por RIVERA (2004), para a obtenção dos dados experimentais, as características químicas, físico-hídricas e os parâmetros da curva de retenção de água no solo podem ser conferidos nas Tabelas $1 ; 2$ e 3.

TABELA 1. Características químicas do solo utilizado neste estudo. Chemical characteristics of the soil used in the research.

\begin{tabular}{|c|c|c|c|c|c|c|c|c|c|c|}
\hline $\mathrm{Ph}$ & MO* & $\mathrm{P}$ & $\mathrm{S}$ & $\mathrm{K}^{+}$ & $\mathrm{Ca}^{2+}$ & $\mathrm{Mg}^{2+}$ & $\mathrm{H}+\mathrm{Al}^{3+}$ & CTC & V & $\mathrm{m}$ \\
\hline$\left(\mathrm{CaCl}_{2}\right)$ & $\mathrm{g} \mathrm{kg}^{-1}$ & & & \multicolumn{5}{|c|}{-------------------m mol $\mathrm{dm}^{-3}$--------------- } & \multicolumn{2}{|c|}{------ \% $\%$-------- } \\
\hline 4,3 & 7 & 2 & 4 & 1,1 & 9 & 4 & 18 & 32,1 & 44 & 18 \\
\hline
\end{tabular}

*Matéria orgânica

TABELA 2. Características físico-hídricas do solo utilizado neste estudo. Hydro-physical characteristics of the soil used in the research.

\begin{tabular}{|c|c|c|c|c|c|c|}
\hline \multicolumn{3}{|c|}{ Textura } & Den & sidade & \multirow{2}{*}{$\begin{array}{c}\text { Porosidade } \\
(\%)\end{array}$} & \multirow{2}{*}{$\begin{array}{c}\mathrm{Ko} \\
\left(\mathrm{cm} \mathrm{h}^{-1}\right)\end{array}$} \\
\hline Areia (\%) & Silte $(\%)$ & Argila (\%) & Global $\left(\mathrm{g} \mathrm{cm}^{-3}\right)$ & Partículas $\left(\mathrm{g} \mathrm{cm}^{-3}\right)$ & & \\
\hline 67 & 6 & 27 & 1,37 & 2,41 & 43,195 & 10,221 \\
\hline
\end{tabular}

TABELA 3. Parâmetros da curva de retenção, segundo o modelo de GENUCHTEN (1980). Parameters of the soil water retention curve by GENUCHTEN model (1980).

\begin{tabular}{cccccc}
\hline \multicolumn{5}{c}{ Parâmetros da Curva de Retenção } \\
\hline$\theta \mathrm{r}\left(\mathrm{cm}^{3} \mathrm{~cm}^{-3}\right)$ & $\theta \mathrm{s}\left(\mathrm{cm}^{3} \mathrm{~cm}^{-3}\right)$ & $\alpha\left(\mathrm{cm}^{-1}\right)$ & $\mathrm{n}($ adimensional $)$ & $\mathrm{m}$ (adimensional) & $\mathrm{r}^{2}$ \\
\hline 0,113 & 0,482 & 0,029428 & 1,828069 & 0,452975 & 0,987 \\
\hline
\end{tabular}

RIVERA (2004) determinou os parâmetros de transporte de potássio no bulbo úmido a partir da elaboração de curva de eluição ("breakthrough curve"), sendo utilizado como soluto o cloreto de potássio, com concentração de potássio de $500 \mathrm{mg} \mathrm{L}^{-1}$. A aplicação foi feita em coluna de solo inserida em um tubo de PVC de $5 \mathrm{~cm}$ de diâmetro e $20 \mathrm{~cm}$ de altura. Nas amostras coletadas, determinou-se em laboratório o teor de potássio por fotometria de chama. Os volumes de solução coletados foram de $15 \mathrm{~mL}$, o que representava aproximadamente 0,082 volumes de poros. Esse volume foi controlado com balança de precisão de $0,01 \mathrm{~g}$, assumindo-se que a solução tivesse densidade igual a um. Os teores de potássio obtidos foram utilizados como dados de entrada ao programa CXTFIT (desenvolvido pelo U.S. Salinity Laboratory-USDA-Riverside-CA, TORIDE et al., 1999), para determinar os valores dos parâmetros de transporte de solutos no solo: o coeficiente de dispersão-difusão (D), a velocidade da água nos poros (V), a dispersividade $(\lambda)$ e o fator de retardamento (Fr) (Tabela 4$)$.

TABELA 4. Parâmetros de transporte do potássio obtidos com o modelo CXTFIT para a coluna de solo utilizada nesta pesquisa. Transport parameters of potassium in soil column obtained by the model CXTFIT used in the research.

\begin{tabular}{cccc}
\hline \multicolumn{4}{c}{ Parâmetros de Transporte do Potássio } \\
\hline $\mathrm{V}\left(\mathrm{cm} \mathrm{min}^{-1}\right)$ & $\mathrm{D}\left(\mathrm{cm}^{2} \mathrm{~min}^{-1}\right)$ & Fr (adimensional $)$ & $\lambda(\mathrm{cm})$ \\
\hline 1,489 & 2,705 & 4,730 & 1,816655 \\
\hline
\end{tabular}

\section{Análise de sensibilidade}

Diante da obtenção desses parâmetros (físico-hídricos e parâmetros de transporte do soluto no solo), foram aplicados acréscimos positivos e negativos nos seguintes parâmetros: condutividade hidráulica do solo saturado, umidade inicial e saturada do solo, dispersividade, fator de retardamento, vazão do emissor e concentração do potássio. O objetivo foi efetuar análise de sensibilidade, com o intuito de estudar o comportamento do modelo frente às mudanças desses respectivos parâmetros de entrada. 
As variações dos parâmetros de entrada do modelo foram realizadas tomando-se o resultado de 36 pontos em cada simulação e seguindo acréscimos positivos $(+10 \%,+20 \%,+30 \%,+40 \%$, $+50 \%,+60 \%,+70 \%,+80 \%,+90 \%)$ e negativos $(-10 \%,-20 \%,-30 \%,-40 \%,-50 \%,-60 \%,-70 \%$, $80 \%,-90 \%)$. (eq.6):

Para medir o efeito dessas variações nas simulações, determinou-se o parâmetro erro-padrão

$$
e=\sqrt{\frac{\sum_{i=1}^{N}\left(y_{m}(i)-y_{s}(i)\right)^{2}}{N}}
$$

em que,

e - erro-padrão;

$\mathrm{y}_{\mathrm{m}}(\mathrm{i})$ - valor-padrão simulado pelo modelo;

$\mathrm{y}_{\mathrm{s}}(\mathrm{i})$ - valor simulado pelo modelo, e

$\mathrm{N}$ - número de dados da simulação.

\section{RESULTADOS E DISCUSSÃO}

\section{Análise de sensibilidade em relação à distribuição da umidade no bulbo}

O conteúdo de umidade do bulbo é muito sensível a variações da umidade de saturação do solo, porém, quando esse parâmetro começa a decrescer (como conseqüência de redução da porosidade), as células próximas ao emissor ficam saturadas e começa a se formar uma lâmina de água na superfície do solo, aspecto esse que deixa de ser considerado pelo modelo. Em relação à condutividade hidráulica do solo saturado, verifica-se que essa tem efeito relevante sobre a umidade do bulbo, principalmente quando variada negativamente; já para incrementos positivos, a curva do erro-padrão teve menor declividade, sendo, portanto, o modelo menos sensível a incrementos da condutividade hidráulica do que as reduções nesse parâmetro (Figura 4).

Não foi executada a análise de sensibilidade à condutividade hidráulica do solo não-saturado, pois essa não é parâmetro de entrada do modelo, uma vez que é calculada durante a simulação, pois varia dentro do bulbo molhado.

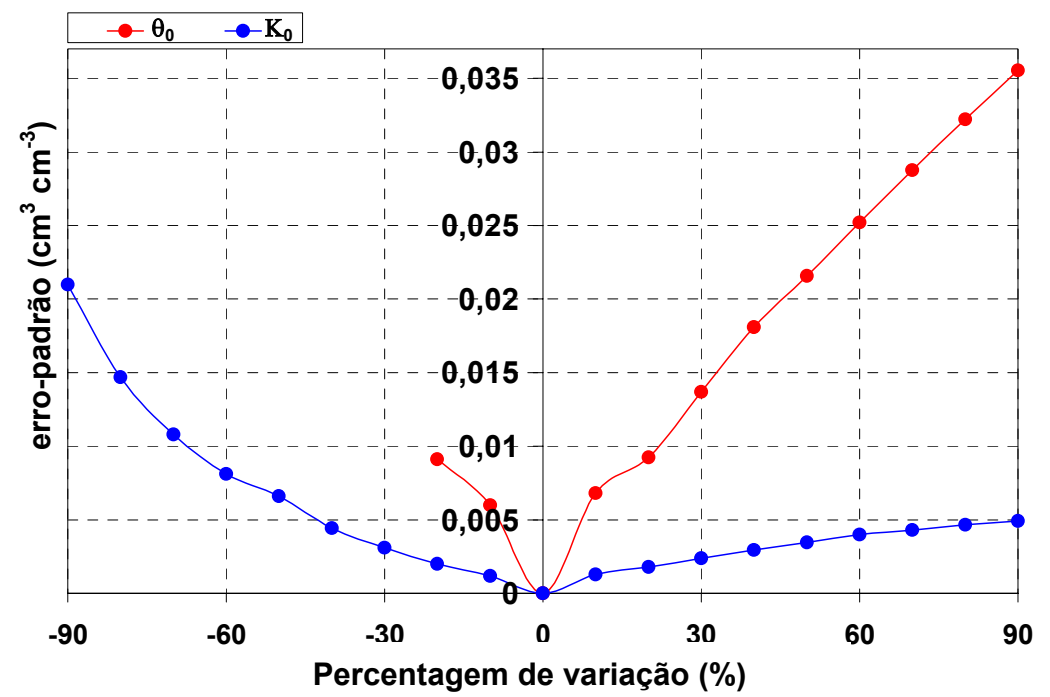

FIGURA 4. Representação da análise de sensibilidade do modelo em relação ao perfil de umidade do solo no bulbo, aplicando de $-90 \%$ a $+90 \%$ de variação na umidade volumétrica de saturação $(\theta o)$ e condutividade hidráulica do solo saturado (Ko). Sensitivity analysis of the model in relation to the soil moisture profile in the bulb, applying variations from $-\mathbf{9 0 \%}$ to $+\mathbf{9 0 \%}$ in the volumetric soil moisture (saturation point, $\theta 0)$ and hydraulic conductivity of saturated soil (Ko) values. 
No caso da umidade inicial do solo (Figura 4), houve limitações na realização da análise de sensibilidade, pois verificou-se que o modelo é muito sensível, tanto a variações positivas quanto a variações negativas desse parâmetro. No primeiro caso, o solo fica rapidamente saturado e logo encharcado na superfície, e no segundo, a umidade do solo pode ficar menor que a umidade residual, aspecto que não pode ser contabilizado pelo modelo por não ter significado físico.

A mudança na vazão, em taxas iguais ou menores que a capacidade de infiltração do solo, acarretou maior erro-padrão quando foi decrescida; quando acrescida, os erros-padrão foram bem menores, ou seja, o modelo foi mais sensível a reduções da vazão da fonte pontiforme do que a incrementos (Figura 5).

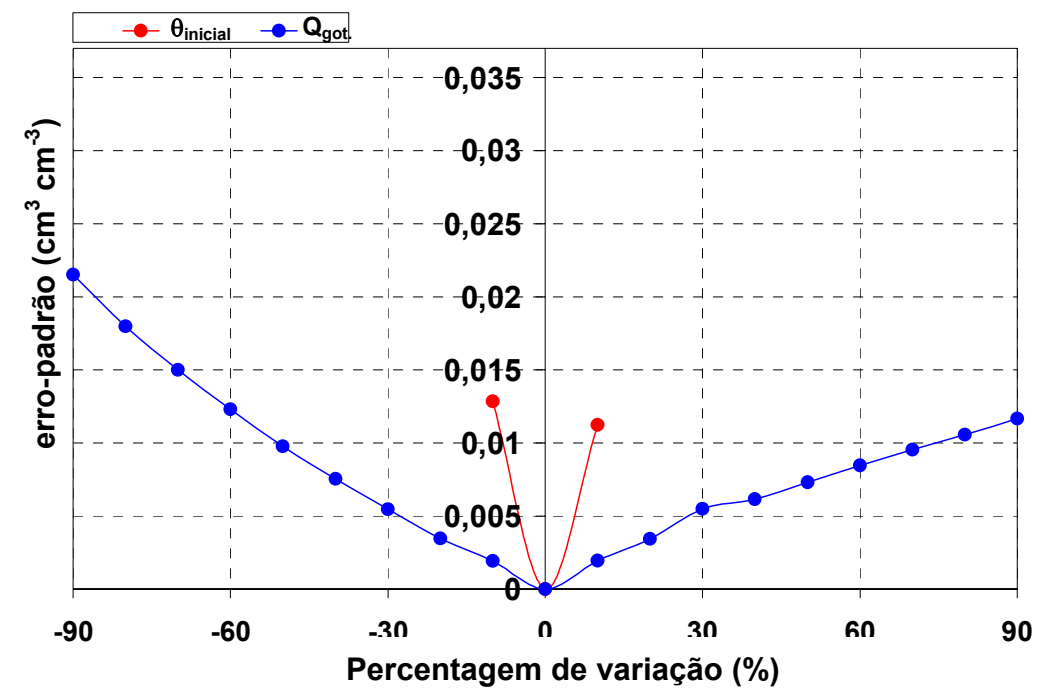

FIGURA 5. Representação da análise de sensibilidade do modelo em relação ao perfil de umidade do solo no bulbo, aplicando de -90 a $+90 \%$ de variação na vazão do gotejador e na umidade volumétrica inicial do solo. Sensitivity analysis of the model in relation to the soil moisture profile in the bulb, applying variations from $-90 \%$ to $+90 \%$ in the flow emitter $\left(Q_{\text {got. }}\right)$ and the initial soil moisture $\left(\theta_{\text {inicial }}\right)$ values.

\section{Análise de sensibilidade em relação à distribuição da concentração do potássio no bulbo}

É pequena a sensibilidade do modelo a incrementos da umidade de saturação do solo ( $\theta 0)$ relacionado com a distribuição do potássio. Já para diminuições de $\theta 0$, o comportamento é similar, mas até certo limite, pois o solo começa a se encharcar, acarretando as impossibilidades já discutidas. A sensibilidade do modelo a respeito desse parâmetro foi menor que para o caso da distribuição da umidade no bulbo, pois o potássio não segue exatamente a mesma dinâmica da água no solo. No que se refere à condutividade hidráulica do solo saturado, o modelo é sensível a decréscimos desse parâmetro, principalmente para valores muito baixos. Para variações positivas, o modelo é pouco sensível, sendo quase que praticamente insensível para valores elevados de Ko (Figura 6).

A sensibilidade do modelo a respeito da umidade inicial do solo teve o mesmo comportamento observado na análise anterior, ou seja, o modelo foi muito sensível na faixa de -10 a $+10 \%$; no intervalo restante, ocorreu impossibilidade de cálculo, pois ou o solo ficava encharcado na superfície ou a umidade do solo ficava menor que a umidade residual.

Observou-se que a vazão é um parâmetro que influencia muito na distribuição do potássio no solo, principalmente para variações negativas, quando o modelo demonstrou ser muito sensível, pois como esse cátion se desloca fundamentalmente por fluxo de massa, baixas vazões afetam sensivelmente sua movimentação, diminuindo seu transporte. Já para incrementos desse parâmetro, a sensibilidade foi moderada (Figura 7). 
Com relação às características que relacionam soluto e solo, a dispersividade foi o fator que menor sensibilidade provocou no modelo, possivelmente pelo fato de que a contribuição do fluxo por dispersão hidrodinâmica ao deslocamento no potássio no solo é baixa, tal como relatado por VOGELER et al. (1997).

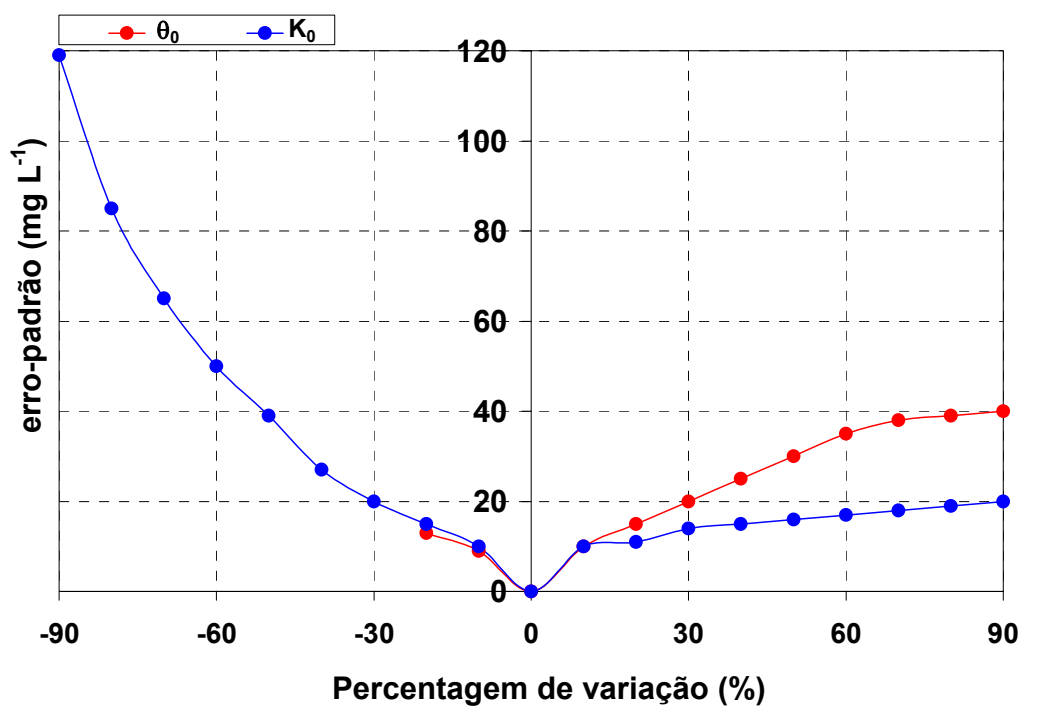

FIGURA 6. Representação da análise de sensibilidade do modelo em relação à concentração do potássio no bulbo, aplicando de -90 a $+90 \%$ de variação na umidade volumétrica de saturação $(\theta o)$ e na condutividade hidráulica do solo saturado (Ko). Sensitivity analysis of the model in relation to the potassium concentration in the bulb, applying variations from $-\mathbf{9 0 \%}$ to $+\mathbf{9 0 \%}$ in the volumetric soil moisture (saturation point, $\theta 0$ ) and hydraulic conductivity of saturated soil (Ko) values.

Já no caso do fator de retardamento, os maiores valores do erro-padrão foram obtidos quando foi realizado decréscimo nos valores desse parâmetro (com valor máximo para a redução de 90\%), possivelmente devido ao fato de que, conforme esse parâmetro diminui, o transporte do potássio tende a ser maior.

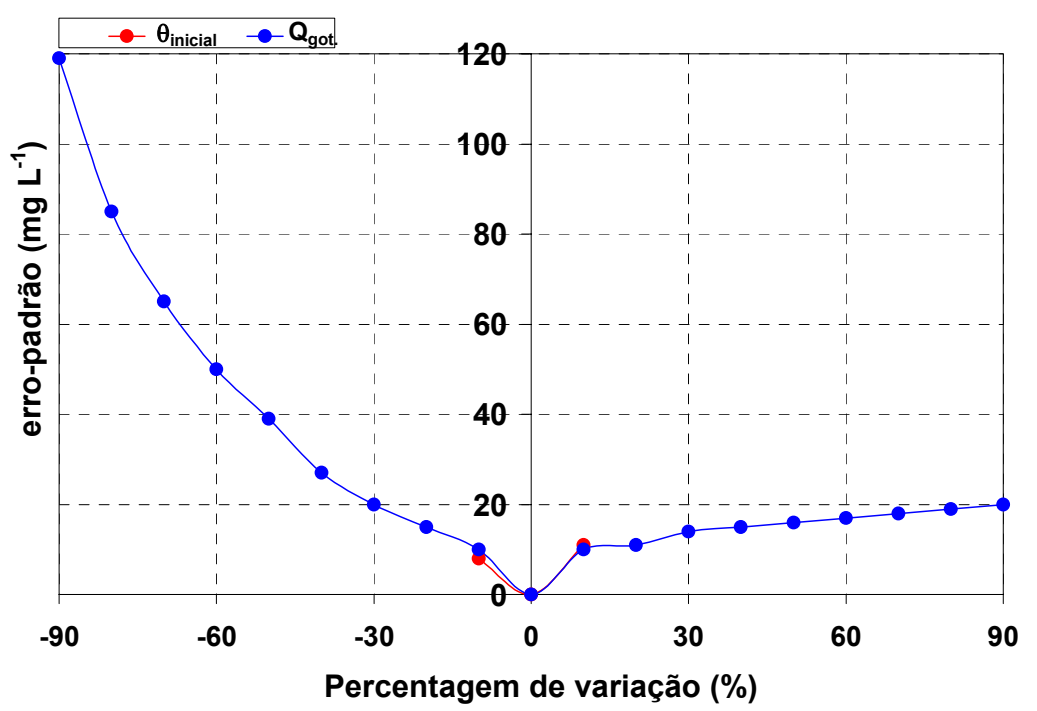

FIGURA 7. Representação da análise de sensibilidade do modelo em relação à concentração do potássio no bulbo, aplicando de -90 a $+90 \%$ de variação na vazão do gotejador e na umidade volumétrica inicial do solo. Sensitivity analysis of the model in relation to the potassium concentration in the bulb, applying variations from $-90 \%$ to $+90 \%$ in the in the flow emitter $\left(Q_{\text {got. }}\right)$ and the initial soil moisture $\left(\theta_{\text {inicial }}\right)$ values. 
Para variações positivas do fator de retardamento, o modelo foi apenas moderadamente sensível (Figura 8).

Dos parâmetros analisados na distribuição de umidade dentro do bulbo, os que tiveram maior erro-padrão (conseqüentemente revelaram maior sensibilidade do modelo) foram, em ordem decrescente de importância, a umidade inicial e a umidade de saturação do solo, a vazão do emissor e a condutividade hidráulica do solo saturado, principalmente para variações negativas desses dois últimos. Resultados semelhantes foram obtidos por CHRYSIKOPOULOS et al. (1990) e MAGESAN et al. (1995).

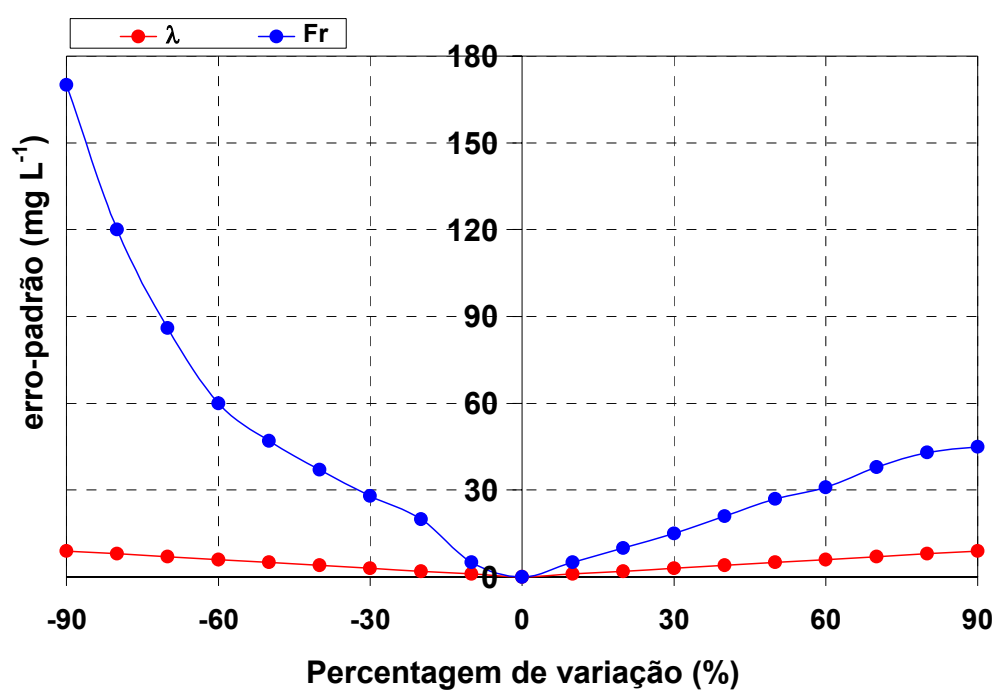

FIGURA 8. Representação da análise de sensibilidade do modelo em relação à concentração de potássio no bulbo, aplicando de -90 a $+90 \%$ de variação na dispersividade $(\lambda)$ e no fator de retardamento do solo (Fr). Sensitivity analysis of the model in relation to the potassium concentration in the bulb, applying variations from $-90 \%$ to $+90 \%$ in the dispersivity $(\lambda)$ and retardation factor $(\mathrm{Fr})$ values.

Na distribuição do potássio, o modelo é mais sensível, em ordem decrescente de prioridade, a diminuição do valor do fator de retardamento, da vazão do gotejador e da condutividade hidráulica do solo saturado.

Para que o presente modelo possa ser aplicado em condições de campo, é necessária a inclusão de rotina que contabilize a extração de água e de solutos pelas plantas. Essa é a próxima etapa de desenvolvimento a que se tem dedicado a equipe executora deste trabalho.

\section{CONCLUSÕES}

O modelo, no que diz respeito à distribuição da água no solo, mostrou-se bastante sensível a variações da umidade de saturação e da umidade inicial do solo e moderadamente sensível a decréscimos da vazão do emissor e da condutividade hidráulica do solo saturado.

A distribuição do potássio é afetada fundamentalmente pelas propriedades hidráulicas e físico-químicas do solo e pela taxa de aplicação de água no solo, sendo o modelo bastante sensível a variações negativas da condutividade hidráulica do solo saturado, do fator de retardamento do solo e da vazão do gotejador.

O modelo mostrou-se pouco sensível às variações da dispersividade do solo, demonstrando que, nas condições testadas, o potássio desloca-se principalmente pelo fluxo de massa. 


\section{REFERÊNCIAS}

ALVAREZ, J.; HERGUEDAS, A.; ATIENZA, J. Modelización numerica y estimación de parámetros para la descripción del transporte de solutos en colunas de suelo en laboratorio. Madrid: INIA, 1995. 69 p.

BOTREL, T.A. Simulação da distribuição espacial da água em solo irrigado com gotejador. 1988. 80 f. Tese (Doutorado em Solos e Nutrição de Plantas) - Escola Superior de Agricultura "Luiz de Queiroz", Universidade de São Paulo, Piracicaba, 1988.

CHRYSIKOPOULOS, C.V.; KITANDIS, P.K.; ROBERTS, P.V. Analysis of one-dimensional solute transport through porous media with spatially variable retardation factor. Water Resources Research, Washington, v.26, n.3, p.437-46, 1990.

CLOTHIER, B.E. Solute travel times during trickle irrigation. Water Resources Research, Washington, v.20, n.12, p.1.848-52, 1984.

CRUZ, R.L. Modelização do balanço hídrico de uma cultura irrigada por um sistema de irrigação localizada. 2000. 80 f. Tese (Livre-Docência em Irrigação e Drenagem) - Faculdade de Ciências Agronômicas, Universidade Estadual Paulista, Botucatu, 2000.

JURY, W.A. Field scale water and solute transport through unsatured soils. In: SAHINBERG, I.; SHALHEVET, J. Soil salinity under irrigation: Process and management. Amsterdam: SpringVerlag, 1984. p.115-25.

LIBARDI, P.L. Dinâmica da água no solo. São Paulo: Editora da Universidade de São Paulo, 2005. $335 \mathrm{p}$.

MAGESAN, G.N.; VOGELER, I.; SCOTTER, D.R.; CLOTHIER, E.; TILLMAN, R.W. Solute movement through two unsaturated soils. Australian Journal Soil Research, Austrália, v.33, p.58596, 1995.

MATOS, A.T.; COSTA, L.M.; FONTES, M.P.; MARTINEZ, M.A. Retardation factors and the dispersion-diffusion coefficients of $\mathrm{Zn}, \mathrm{Cd}, \mathrm{Cu}$ and $\mathrm{Pb}$ in soils from Viçosa-MG, Brazil. Transactions of the ASAE, St. Joseph, v.42, n.4, p.903-10, 1999.

MMOLAWA, K.; OR, D. Root zone solute dynamics under drip irrigation: a review. Plant and Soil, Wageningen, v.22, n.2, p.163-90, 2000.

PREVEDELLO, C.L. Física de solos com problemas resolvidos. Curitiba: Saeafs, 1996. 446 p.

RIVERA, R.N.C. Modelagem da dinâmica da água e do potássio na irrigação por gotejamento superficial. 2004. 89 f. Tese (Doutorado em Irrigação e Drenagem) - Escola Superior de Agricultura "Luiz de Queiroz", Universidade de São Paulo, Piracicaba, 2004.

RIVERA, R.N.C.; DUARTE, S.N.; MIRANDA, J.H.; BOTREL, T.A. Modelagem da dinâmica do potássio no solo sob irrigação por gotejamento: validação do modelo. Engenharia Agrícola, Jaboticabal, v.26, n.2, p.388-94, 2006.

TORIDE, N.; LEIJ, F.; GENUCHTEN, M. TH. Van. The CXTFIT code for estimating parameters from laboratory or field tracer experiments, verion 2.1. Califórnia: Research Report U.S./Salinity Laboratory Agricultural Research Service/U.S. Departament of Agriculture, 1999. 85 p.

TULI, A.; HOPMANS, J.W. Effect of degree of fluid saturation on transport coefficients in disturbed soils. European Journal of Soil Science, United Kingdom, v.55, n.1, p.147-64, 2004.

VAN DER PLOEG, R.R.; BENECKE, P. Unsteady unsatured, n-dimensional moisture flow in soil: a computer simulation program. Soil Science Society of America Proceedings, Madison, v.38, n.6, p.881-5, 1974.

GENUCHTEN, M. TH. Van. A closed form equation for predicting the hydraulic conductivity of unsatured Soils. Soil Science Society American Journal, Madison, v.44, n.4, p.892-8, 1980. 
GENUCHTEN, M. TH. Van; WIERENGA, P.J. Solute dispersion coefficients and retardation factors. In: KLUTE, A. Methods of soil analysis. I - Physical and mineralogical methods. Madison: Soil Science Society of America, 1986. p.1.025-54.

VOGELER, I.; SCOTTER, D.R.; CLOTHIER, B.E.; TILLMAN, R.W. Cation transport during unsaturated flow through two intact soils. European Journal of Soil Science, United Kingdom, v.48, n.3, p.401-10, 1997.

WAGENET, R.J. Water and solute flux. In: KLUTE, A. Methods of soil analysis. I - Physical and mineralogical methods. Madison: Soil Science Society of America, 1986. p.1055-88. 